\title{
Métodos empleados y motivos para dejar de fumar de los exfumadores
}

\author{
Elena Samarin Ocampos ${ }^{a}$, Beatriz Gómez Molina ${ }^{a}$, Alex Villalobos Uriola , María Luisa \\ Botello Cruz ${ }^{a}$, Judith Olguín Ramíreza y Francisco López de Castro ${ }^{b}$
}

\begin{abstract}
${ }^{a}$ Residente de Medicina de Familia y Comunitaria. Unidad Docente Multiprofesional de Toledo. 'Médico de Familia. Subdirección de Calidad, Investigación y Docencia del Servicio de Salud de Castilla-La Mancha.
\end{abstract}

Correspondencia:

Elena Samarín Ocampos. Unidad Docente

Multiprofesional de Atención

Familiar y Comunitaria de Toledo.

Correo electrónico: adebacle@hotmail.com

Recibido el 25 de febrero de 2012.

Aceptado para su publicación el 5 de marzo de 2014.

\section{RESUMEN}

Objetivo: conocer el proceso de deshabituación, los métodos empleados para dejar de fumar y los motivos para ello de los exfumadores.

Diseño: estudio observacional descriptivo, basado en entrevistas personales.

Emplazamiento: área de salud de Toledo.

Participantes: exfumadores mayores de 18 años.

Mediciones principales: se recogieron variables sociodemográficas, de consumo de tabaco (edad de inicio, índice tabáquico, intentos de abandono y duración de la abstinencia), métodos de deshabituación utilizados (farmacológicos o no), motivo principal del abandono, problemas tras el abandono, grado de esfuerzo e intención de volver a fumar.

Resultados: muestra final de 393 exfumadores (59,8 \% varones), con edad media de 51,3 años (DE 13,7). La edad media de inicio del consumo de tabaco fue 16,4 años $(3,7)$ y la de abandono 42,4 años (12,9). El 54,7 \% (IC95 \%: 49,8-59,6) había realizado algún intento previo de deshabituación. El fármaco más utilizado fue la nicotina (8,1\%). El 72,5\% (IC95 \%: 68,1-76,9) no utilizó nunca ningún método. Esta cifra es del 78,9 \% en el último intento. Las variables sexo (varón), tiempo de abstinencia (>10 años) e índice tabáquico $(<30)$ son factores de riesgo de dejar de fumar sin ayuda. El motivo fundamental que alegan para dejar de fumar es la prevención de enfermedades $(35,2 \%)$ y la presencia de problemas físicos $(26,3 \%)$. Al $32,7 \%$ de los entrevistados dejar de fumar no le supuso ningún esfuerzo. Un 48,8 \% reconoce haber tenido algún problema tras dejar de fumar. El 63,4\% no echa de menos el tabaco.

Conclusiones: El abandono del hábito se produce tras 26 años de consumo y varios intentos. La mayoría no utilizó ningún método para abandonar el hábito. Los motivos tienen que ver sobre todo con la presencia de problemas físicos y la prevención de enfermedades.

Palabras clave: Tabaquismo. Deshabituación. Exfumador. Atención Primaria.

\section{ABSTRACT}

Methods used and former smokers' reasons for giving up smoking

Objective: to establish the process involved in breaking the smoking habit, the methods used to give up smoking and former smokers' reasons for doing so.

Design: observational study based on face-to-face interviews.

Location: Toledo healthcare area.

Participants: former smokers over 18 years of age.

Main analysed data: socio-demographic variables in tobacco consumption, (starting age, smoking index, attempts at giving up and duration of abstinence), habit-breaking methods used (whether pharmacological or not), main reason for giving up, degree of effort and intention to smoke again.

Results: final sample of 393 former smokers (59.8\% male), average age 51.3 years (DE 13.7). The average starting age for tobacco consumption was 16,4 years (3.7) and 42,4 years (12.9) for giving up. 54.7\% (IC95\%: 49,8-59,6) had made a previous attempted at giving up. Th most widely used drug was nicotine (8,1\%). $72.5 \%$ (IC95\%: 68,1-76,9) never used any specific method. This figure is $78.9 \%$ at the last attempt. Variables in sex (male), time of abstinence ( $>10$ years) and smoking index $(<30)$ are risk factors in stopping smoking. The main reasons given for breaking the habit are disease prevention (35.2\%) and the presence of physical problems $(26.3 \%)$. In the case of $32.7 \%$ of those interviewed, no effort was required to cease smoking. $48.8 \%$ acknowledge experiencing some problems on giving it up. $63.4 \%$ do not miss the smoking habit. Conclusions: smoking cessation occurs after 26 years and several attempts. The majority did not use any specific method to give up the habit. Reasons are related above all to the presence of physical problems disease prevention.

Key words: Smoking. Smoking cessation. Ex-smoker. Primary Health Care. 


\section{INTRODUCCIÓN}

El tabaquismo es considerado, tras la hipertensión arterial, el segundo factor de riesgo general conocido que más mortalidad causa en el mundo ${ }^{1}$. Según la OMS, el consumo de tabaco mata a casi seis millones de personas cada año en todo el mundo; si no se toman medidas urgentes, esta cifra podría ascender a ocho millones en $2030^{2,3}$.

En nuestro país, donde la prevalencia del consumo de tabaco, según la Encuesta Nacional de Salud del año 2011/12, es del $24 \%$ de la población ${ }^{4}$, el consumo de tabaco es la primera causa de enfermedad, invalidez y muerte evitable, habiéndose producido, en 2006, más de 53.000 muertes atribuibles al mismo ${ }^{5}$.

Diversos estudios realizados en Estados Unidos y Europa demuestran que cerca de un $70 \%$ de los fumadores activos desean abandonar el hábito tabáquico, pero la gran mayoría de ellos refiere grandes dificultades en conseguirlo $\sin$ ayuda $^{6}$. Estas dificultades están estrechamente ligadas a las propiedades adictivas de la nicotina, ya que es una sustancia capaz de generar gran dependencia, incluso comparable a otras drogas como la cocaína y la heroína ${ }^{7-9}$.

Todas estas afirmaciones remarcan la necesidad de medidas de intervención efectivas sobre los fumadores, en las que el fumador sea contemplado como un enfermo susceptible de intervenciones farmacológicas y conductuales que le lleven a superar su adicción ${ }^{10}$. Se hace por ello importante conocer el proceso de deshabituación y los motivos que convencen al fumador para abandonar su hábito. En este sentido, la experiencia de los exfumadores puede proporcionarnos una valiosísima información sobre ello, pudiendo incluso suponer una herramienta más para poder orientar y lograr la deshabituación de los fumadores activos ${ }^{11}$.

La mayoría de los estudios existentes sobre la deshabituación tabáquica y el método empleado para conseguirlo se basan en pacientes fumadores en activo que quieren dejar de fumar y abarcan un seguimiento máximo de un año de los mismos ${ }^{12,13}$. La opinión de los exfumadores consolidados y las herramientas definitivas que han sido utilizadas para dejar de fumar no han sido investigados aún en profundidad.

En este contexto, nos planteamos realizar este estudio, cuyos objetivos fueron conocer el proceso de deshabituación seguido hasta llegar a la condición de exfumador, los métodos empleados para dejar de fumar y los motivos que hicieron abandonar el hábito tabáquico a los exfumadores.

\section{MATERIAL Y MÉTODOS}

Se trata de un estudio observacional descriptivo, basado en entrevista personal, realizado en el área de salud de Toledo, entre los meses de marzo y abril de 2013.

La población del estudio eran las personas mayores de 18 años que hubieran fumado durante al menos un año y llevaran más de un año sin fumar (según definición de exfumador de la OMS) ${ }^{14}$.

La muestra se recogió por reclutamiento en las salas de espera de los centros de salud de Buenavista, Sillería, Palomarejos, Santa María de Benquerencia, Polán, Santa Bárbara y Bargas, en el período arriba citado. El tamaño muestral necesario para hacer una estimación de porcentajes $(p=50$ $\%$ ) con error alfa 0,05 y precisión $\pm 5 \%$, fue de 403 casos.

Mediante entrevista personal, realizada por los propios investigadores, se recogieron las siguientes variables:

- Sociodemográficas: edad, sexo, nivel de estudios (básicos, secundarios y universitarios) y nacionalidad.

- Variables relativas al consumo de tabaco: edad de inicio, años de consumo, cantidad de cigarrillos/día, índice tabáquico [IT] ( $\mathrm{n}^{\circ}$ cigarrillos / día $\times n^{\circ}$ años /20), intentos de abandono, edad de abandono y duración de la abstinencia.

- Métodos de deshabituación utilizados alguna vez (en intentos previos) y en el último intento, diferenciando farmacológicos (bupropion, nicotina y vareniclina) o no farmacológicos (psicoterapia, acupuntura, hipnosis u otros).

- Motivo principal del abandono (pregunta abierta).

- Valoración del grado de influencia de los siguientes motivos: aparición de problemas físicos, prohibición de fumar en lugares públicos, embarazo, presión familiar, cuestiones estéticas, papel ejemplar para los hijos, gasto económico, consejo del personal sanitario, sentimiento personal de ser dependiente del tabaco, imágenes y mensajes en cajetillas de tabaco.

- Problemas tras el abandono del tabaco: ganancia de peso, nerviosismo y otros.

- Grado de esfuerzo realizado para dejar de fumar, añoranza del hábito e intención de volver a fumar. 
Antes de iniciar la recogida de datos se realizó un pilotaje con las primeras 50 personas captadas, a raíz del cual se hicieron pequeñas modificaciones en el cuestionario.

Los datos fueron introducidos en una base del programa SPSS 12.0 para Windows, que se empleó también para la descripción y análisis estadístico. Se utilizaron las herramientas descriptivas habituales (media, porcentajes y desviaciones), calculándose los intervalos de confianza $95 \%$ de las variables principales del estudio. Las pruebas de análisis empleadas fueron $\chi^{2}$ de Pearson y $U$ de Mann-Whitney. Se realizó una regresión logística binaria (método hacia adelante) para valorar la existencia de variables de confusión en el análisis de asociaciones.

\section{RESULTADOS}

Se solicitó la colaboración de 408 individuos; rechazando cinco de ellos participar en el estudio. Diez cuestionarios se descartaron posteriormente por no incluir toda la información o no cumplir los criterios de inclusión, resultando una muestra final de 393 (59,8\% varones y 40,2\% mujeres), con una edad media de 51,3 años (DE 13,7). El 97,2 $\%$ eran de nacionalidad española, con la siguiente distribución del nivel de estudios: básicos, 33,6 \%; secundarios, 33,6 \% y universitarios, 32,8 \%.
La edad media de inicio del consumo de tabaco fue 16,4 años (DE 3,7) y la de abandono 42,4 años (DE 12,9). El índice tabáquico medio fue de 30,1 paquetes/año (DE 28,0). El tiempo medio sin fumar (abstinencia) fue de 9,2 años (DE 9,0); el 61,1\% llevaba menos de 10 años sin fumar. El 54,7 \% (IC95 $\%: 49,8-59,6$ ) había realizado algún intento previo de deshabituación tabáquica; esta cifra es superior en exfumadores con un IT $\geq 30$ que en aquellos con consumos inferiores $(64,9$ vs $48,1 \% ; p=0,001)$. El $10 \%$ había realizado más de tres intentos.

En intentos previos de deshabituación, el fármaco más utilizado fue la nicotina $(8,1 \%)$ como puede verse en la figura 1. El 72,5 \% (IC95 \%: 68,1-76,9) no utilizó nunca ningún método. Esta cifra es del $78,9 \%$ si solo se contempla el último intento, siendo en este último caso el método más empleado la vareniclina, en el 6,4\% de los exfumadores. Dejar de fumar sin ayuda en el último intento (tabla 1) resultó significativamente más frecuente en exfumadores con tiempo de abstinencia mayor de 10 años, en los varones y en los exfumadores con menor consumo tabáquico $(\mathrm{IT}<30)$. Por el contrario, la edad, la nacionalidad o el nivel de estudios no resultaron ser factores influyentes para dejar de fumar sin ayuda en el último intento. En el análisis multivariante (tabla 2), se observa como las variables sexo, tiempo de abstinencia e IT se mantienen como factores de riesgo de dejar de fumar sin ayuda.

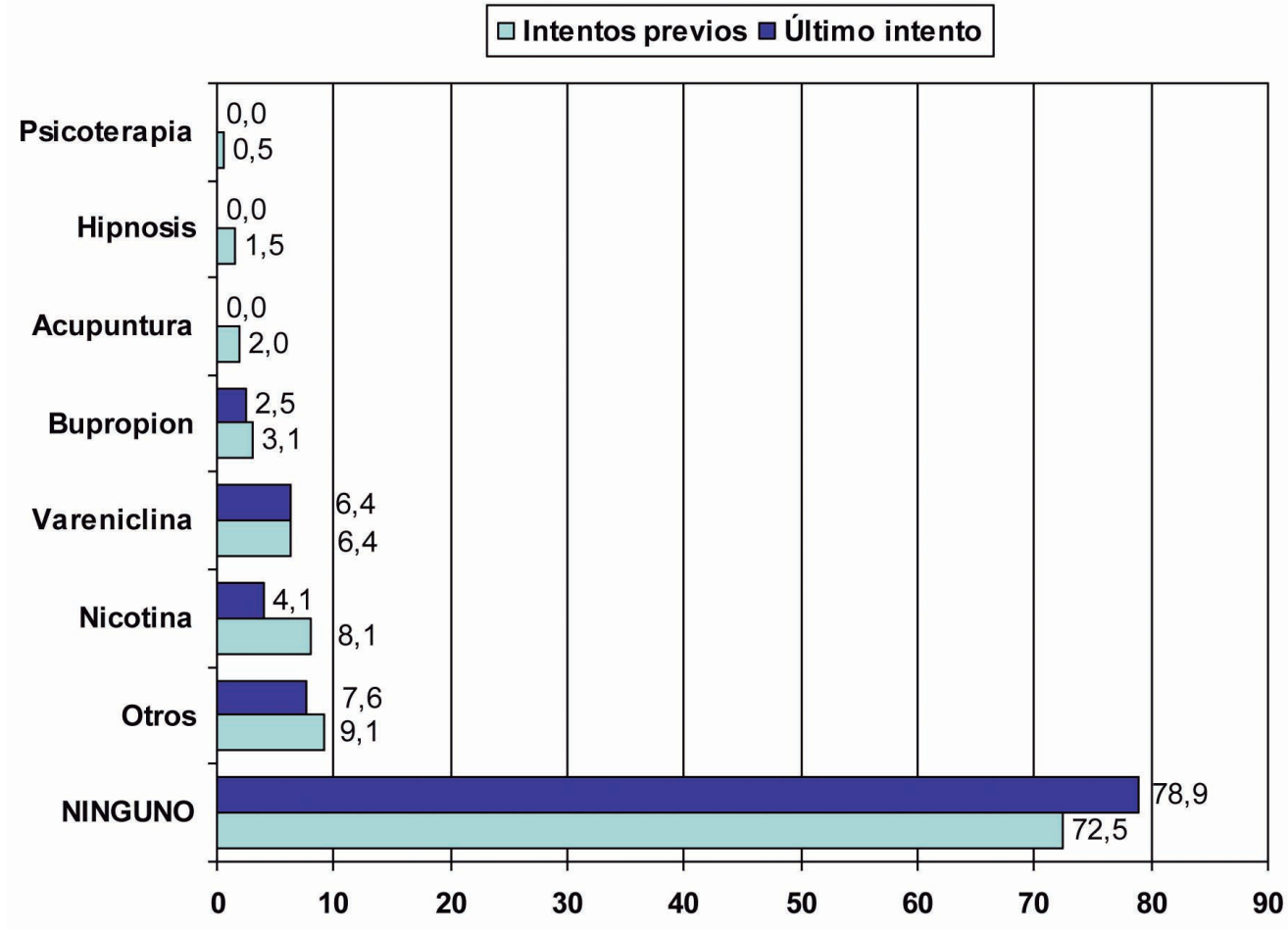

Figura 1. Uso de métodos de deshabituación tabáquica en los intentos previos y en el último intento realizado 


\begin{tabular}{llccc}
\hline Variables & Categorías & Sin ayuda & Con ayuda & p \\
\hline SEXO & Hombre & $82,6 \%$ & $17,4 \%$ & 0,030 \\
& Mujer & $73,4 \%$ & $26,6 \%$ & \\
EDAD & $\leq 50$ años & $77,8 \%$ & $22,2 \%$ & 0,640 \\
ESTUDIOS & $>50$ años & $79,7 \%$ & $20,3 \%$ & \\
& Básicos & $82,6 \%$ & $17,4 \%$ & 0,186 \\
TIEMPO ABSTINENCIA & Secundarios & $80,3 \%$ & $19,7 \%$ & \\
\multirow{2}{*}{ ÍNDICE TABÁQUICO } & Universitarios & $73,6 \%$ & $26,4 \%$ & \\
& $<10$ años & $72,9 \%$ & $27,1 \%$ & 0,000 \\
& $\geq 10$ años & $88,2 \%$ & $11,8 \%$ & \\
\hline
\end{tabular}

Tabla 1: Relación entre sexo, edad, nivel de estudios, tiempo de abstinencia e índice tabáquico con dejar de fumar sin usar ningún método en el último intento

\begin{tabular}{lccccr}
\hline \multirow{2}{*}{ Variables en la ecuación } & Test de Wald & p & OR & \multicolumn{2}{c}{ IC 95\% OR } \\
\cline { 5 - 6 } & & & & Inferior & Superior \\
\hline Sexo (varón) & 6,121 & 0,013 & 1,996 & 1,154 & 3,451 \\
Edad > 50 años & 0,208 & 0,648 & 1,146 & 0,638 & 2,061 \\
Índice tabáquico (<30) & 6,156 & 0,013 & 2,164 & 1,176 & 3,981 \\
Nacionalidad (extranjero) & 0,681 & 0,409 & 2,422 & 0,296 & 19,807 \\
Abstinencia (10 ó más años) & 8,376 & 0,004 & 2,405 & 1,328 & 4,358 \\
Constante & 9,853 & 0,002 & 0,031 & & \\
\hline
\end{tabular}

Tabla 2. Resultados de la regresión logística (método Enter). Variable dependiente: dejar de fumar sin usar ningún método en el último intento. OR: Odds ratio.

En cuanto al motivo fundamental que los exfumadores alegan para dejar de fumar (figura 2), los más frecuentes fueron la "prevención de enfermeda- des" $(35,2 \%)$ y la "presencia de problemas físicos de salud" (26,3\%).

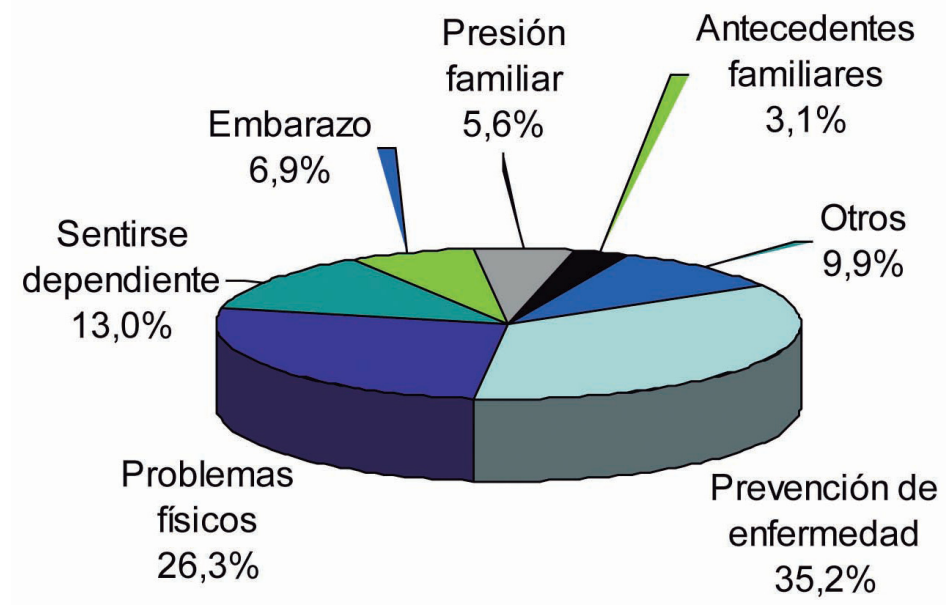

Figura 2: Principales motivos para dejar de fumar 
En la figura 3 se muestra la influencia atribuida a los distintos motivos para dejar de fumar planteados en la encuesta. Se observa cómo los considerados más influyentes por los entrevistados son la presencia de problemas físicos $(32,8 \%$ lo considera muy influyente), el sentimiento personal de dependencia (32,3\%) y el consejo sanitario (22,6 \%). Por el contrario, los considerados menos influyentes fueron las imágenes en las cajetillas de tabaco $(2,8$ $\%)$, las cuestiones estéticas $(4,8 \%)$ y la prohibición de fumar en lugares públicos (4,8 \%). que tener en cuenta no solo que han sido obtenidos a través de encuesta y son, por tanto, subjetivos, sino que, además, en algunos casos se trata de exfumadores con muchos años de abstinencia (más de la mitad llevaban 10 años sin fumar), en quienes el tiempo puede haber "suavizado" el recuerdo de su proceso de deshabituación.

Lo que sí es un hecho objetivo es que el consumo de tabaco es un hábito muy extendido del que, según algunos estudios, es difícil escapar, ${ }^{6,15}$. Esto

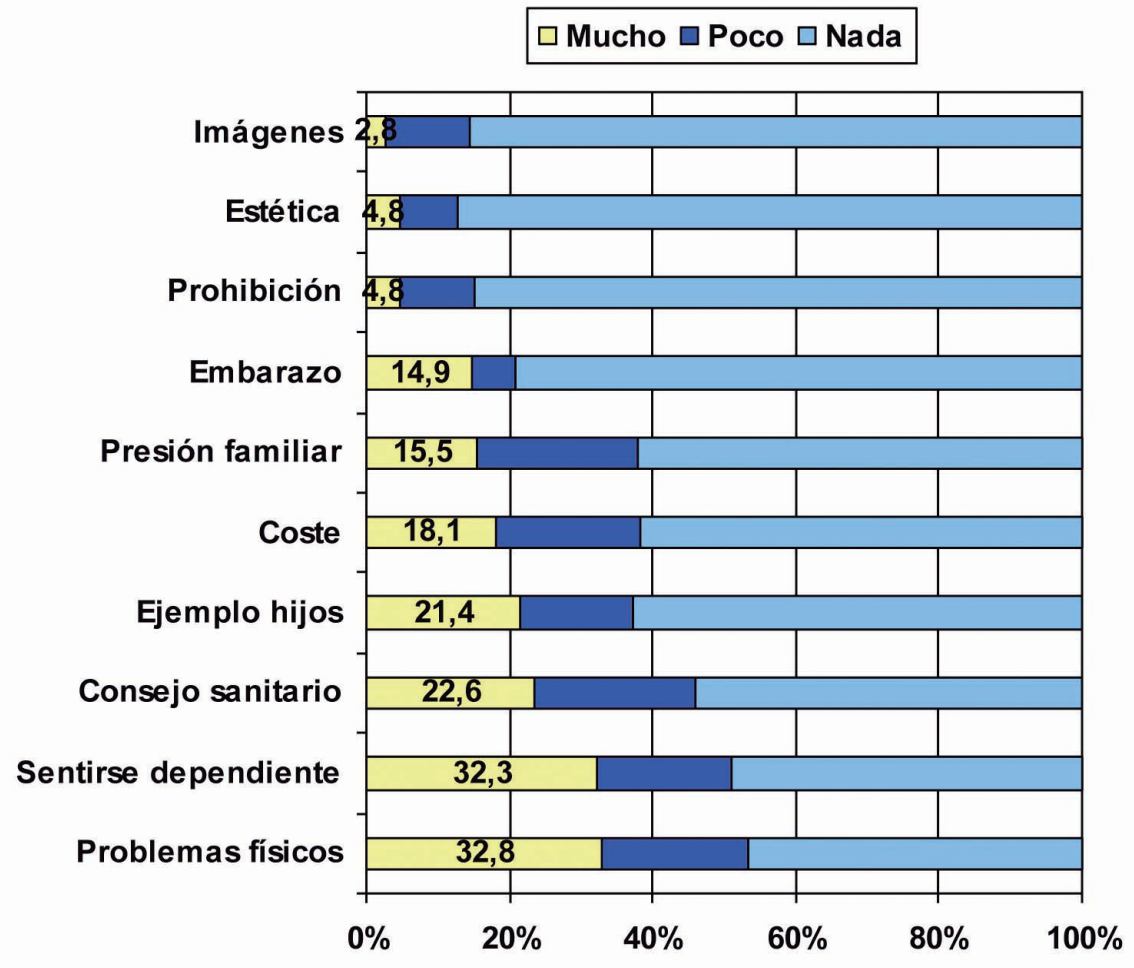

Figura 3. Grado de influencia atribuida a distintos motivos para dejar de fumar planteados en la encuesta
Al 32,7 \% de los entrevistados dejar de fumar no le supuso ningún esfuerzo, al 39,6\%, un poco, y al $27,7 \%$, mucho. Un $48,8 \%$ reconoce haber tenido algún problema tras dejar de fumar. Los más frecuentes fueron el aumento de peso $(39,9 \%)$ y el nerviosismo $(19,4 \%)$.

El $63,4 \%$ no echa de menos el tabaco, el 27,7\% un poco y el $8,9 \%$ mucho. Un 10,0 \% consideran que volverán a fumar. Tanto este porcentaje como la añoranza del tabaco son menores en exfumadores con más de 10 años de abstinencia y mayores de 50 años.

\section{DISCUSIÓN}

Quisiéramos aclarar, antes de iniciar la discusión de nuestros resultados, que para interpretar adecuadamente los datos que hemos presentado hay contrasta, como vemos, con los datos de nuestro estudio en el que, a pesar de que la mitad reconoce haber tenido algún problema tras dejar de fumar, un tercio de nuestros encuestados afirmaron que lo hicieron sin esfuerzo y un $40 \%$ solo con un poco. No hemos encontrado estudios que reflejen resultados similares, por lo que debemos tener en cuenta el posible sesgo tras un periodo de abstinencia de tantos años, y pensar que los resultados reales podrían ser algo peores. Aun así, la opinión de los exfumadores entrevistados sobre la facilidad para dejar de fumar (tal vez cuando ya estaban realmente motivados a dejarlo) puede ser muy útil para transmitir a los fumadores que el abandono del tabaco no es tan difícil como parece. Por eso, puede resultar muy útil incluir a exfumadores en los grupos de apoyo a los fumadores en periodo de deshabituación ${ }^{11}$. 
En ese mismo sentido, uno de los resultados principales de nuestro estudio es que las tres cuartas partes de los exfumadores dejaron de fumar sin ayuda, de forma similar a como reflejan también otros estudios ${ }^{16-19}$. Esto implica que la mayoría lo consigue sin usar ningún método farmacológico o de otro tipo y ello podría servir también de estímulo para aquellos pacientes que se encuentran en fase contemplativa, respaldar el consejo sanitario por parte de los médicos o integrarlo en grupos de ayuda. Todo ello sin olvidar que esto sucede con más frecuencia en fumadores con baja dependencia y que, por tanto, hay un importante colectivo que sí necesitará ayuda. Posiblemente, dejar de fumar sin ayuda puede guardar relación con la motivación real en el momento de iniciar la deshabituación, y ratifica que lo más importante para ayudar a nuestros pacientes es reforzar esa motivación para lograr con éxito dejar de fumar ${ }^{20-22}$. En estudios previos se ha demostrado que las principales causas de fracaso en el periodo de deshabituación han sido la falta de motivación $(24,7 \%)^{12}$.

Entre los motivos más importantes que llevan a los fumadores a decidir dejar de fumar destaca, en más de la mitad de los casos, la presencia de problemas de salud relacionados con el consumo activo de tabaco o el ánimo de prevenirlos, seguido de un sentimiento personal de dependencia. Con porcentajes más bajos se encuentran otros motivos, como el embarazo (sobre todo en mujeres), los antecedentes familiares, la presión familiar o el consejo sanitario. En mayor o menor medida, estos resultados coinciden con lo hallado en otros estudios $^{12,13,15,21}$. Por otro lado, Ilama la atención la escasa influencia atribuida a la prohibición de fumar en lugares públicos, cuando está demostrado que esa medida reduce la prevalencia de tabaquismo y la frecuencia de enfermedades a su consumo ${ }^{23,24}$.

Los datos de la historia de consumo de tabaco reflejada en nuestros encuestados están en consonancia con los resultados de otros estudios ${ }^{15,25,26}$. Hemos de destacar el hecho de que luchamos contra un hábito tóxico que acompaña al fumador durante un promedio de... ¡26 años! Esto debe animarnos a los profesionales sanitarios a insistir en el consejo antitabaco, de forma precoz y sistemática ${ }^{21,27}$, ya que como vemos, es reconocido como efectivo por un amplio grupo de encuestados y ha demostrado su utilidad ${ }^{28-30}$.

Más de la mitad de los exfumadores había realizado algún intento previo de deshabituación, siendo superior en exfumadores con IT $\geq 30$ que en aquellos con menor consumo ${ }^{15}$. Este dato debe de alertarnos, puesto que a mayor IT, mayor es la proba- bilidad de recaída, por lo que deberíamos ser más cuidadosos, dedicarles más tiempo e incidir más en ellos para concienciarles de la importancia de dejar de fumar.

El $70 \%$ de los fumadores entran en contacto con los centros de atención primaria cada año, por lo cual es el lugar desde donde más oportunidades existen para concienciar a los pacientes acerca de su problema y brindarles la ayuda necesaria para conseguirlo. Para ello, según el consenso para la atención clínica al tabaquismo en España, la mejor estrategia es identificar a los fumadores y motivarles para que abandonen el hábito, personalizando los riesgos y costes del consumo de tabaco ${ }^{31}$. También el programa de actividades preventivas y de promoción de la salud (PAPPS) de la Sociedad Española de Medicina de Familia recomienda el consejo claro y personalizado al fumador y el ofrecimiento de ayuda para abandonar el consumo ${ }^{10}$. Además, el médico debe desempeñar un papel modélico, educador, social y terapéutico con los pacientes en proceso de deshabituación, sea cual sea la fase en la que se encuentren ${ }^{21,27}$.

Como conclusión, debemos tratar de transmitir al fumador que dejar de fumar no es tan difícil como parece, que otros lo han conseguido y que, aunque muchas veces no hace falta el uso de fármacos, existen tratamientos eficaces a su disposición, en caso necesario.

\section{BIBLIOGRAFIA}

1. Centro de Información de las Naciones Unidas. Día Mundial sin Tabaco 2012. Disponible en: http://www.cinu.mx/ eventos/observancia/dia-mundial-sin-tabaco-1/ [consultado el 15-07-2013]

2. World Health Organization. Estadísticas Sanitarias Mundiales. 2013. Disponible en: http://www.who.int/gho/publications/world health_statistics/2013/en/indei.html [consultado el 11-02-2014]

3. Mathers CD, Loncar D. Projections of global mortality and burden of disease from 2002 to 2030. PLoS Med. 2006;3(11):e442.

4. Instituto Nacional de Estadística. Encuesta Nacional de Salud 2011-2012. Disponible en: http://www.ine.es/prensa/np770.pdf [consultado el 11-02-2014]

5. Banegas JR, Díez L, Bañuelos B, González-Enríquez J, Villar F, Martín JM, et al. Mortalidad atribuible al consumo de tabaco en España en 2006. Med Clin (Barc). 2011;136:97102.

6. Fiore MC, Baker TB. Treating Smokers in the Health Care Setting. N Engl J Med. 2011;365:1222-31.

7. Rabinoff M, Caskey N, Rissling A, Park C. Pharmacological and chemical effects of cigarette additives. Am J Public Health. 2007;97(11):1981-91. 
8. Muñoz MA, Sanjuán R, Fernández-Santaella MC, Vila J, Montoya P. Aspectos neuropsicológicos del craving por la nicotina. Adicciones. 2011;23 (2),111-23.

9. Fu M, Martínez-Sánchez JM, López MJ, Nebot M, Raich A, Fernández E. Dependencia a la nicotina y preparación para dejar de fumar en la población española. Adicciones. 2011;23(2):103-09.

10. Córdoba R, Cabezas C, Camarelles F, Gómez J, Díaz Herráez D, López A et al. Grupos de expertos del PAPPS. Recomendaciones sobre el estilo de vida. Aten Primaria. 2012;44 Supl 1:16-22.

11. Echer IC, Barreto SS. La determinación y el apoyo como factores de éxito en el abandono del tabaquismo. Rev Lat Am Enfermagem. 2008;16(3):445-51.

12. Ramón Torrell JM, Bruguera Cortada E, Fernández Pinilla C, Sanz de Burgoa V, Ramírez Vázquez E. Motivos para dejar de fumar en España en función del sexo y la edad. Gac Sanit. 2009;23(6):539.e1-6.

13. Vila $A$, Rodríguez $C$, Ochoa $O$, Bria $X$. ¿Por qué dejan de fumar realmente nuestros pacientes? Aten Primaria. 2002;30(8):527.

14. Organización Mundial de la Salud. CIE-10. Trastornos mentales y del comportamiento. Pautas diagnósticas y de actuación en Atención Primaria. Geneva: World Health Organization; 1996.

15. Pascual-Lledó JF, De la Cruz-Amorós E, Bustamante-Navarro R, Buades-Sánchez MR, Contreras-Santos C, Castillo-Aguilar C. Abstinencia de tabaquismo tras 12 meses de seguimiento en una unidad de tabaquismo integral. Med Clin (Barc). 2006;126(16):601-6.

16. Chapman S, MacKenzie R. The global research neglect of unassisted smoking cessation: causes and consequences. PLoS Med. 2010;7(2):e1000216.

17. Konfino J, Ondarsuhu D, Goldberg L, Linetzki B, Caixeta $\mathrm{R}$, Ferrante D. Encuesta mundial de tabaquismo en adultos 2012 : resultados de la primera implementación en Argentina. Rev Arg Salud Pública. 2013;4(16):6-15.

18. Servan-Mori E, Heredia-Pi I, Reynales-Shigeatsu L, Bautista-Arredondo S. Intervenciones para dejar de fumar en Mexico: análisis de disponibilidad a pagar por un método efectivo de cesación. Salud Pública Mex. 2012;54(3):213224.

19. Zhu S, Melcer T, Sun J, Rosbrook B, Pierce JP. Smoking cessation with and without assistance: a population-based analysis. Am J Prev Med. 2000;18: 305-311.

20. Sampablo Lauro I, Carreras JM, Lores L, Quesada M, Coll F, Sánchez Agudo L. Deshabituación tabáquica y bupropión: la ansiedad y la depresión como índices de eficacia terapéutica. Arch Bronconeumol. 2002;38(8):351-5.
21. Torrecilla $M$, Barrueco $M$, Jiménez Ruiz $C$, Maderuelo J, Plaza M, Hernández Mezquita M. El médico y el paciente en la toma de decisión del abandono del tabaco. Repercusión de la iniciativa en el resultado de la intervención. Arch Bronconeumol. 2001;37(3):127-34.

22. Echer IC, Barreto SS. La determinación y el apoyo como factores de éxito en el abandono del tabaquismo. Rev Lat Am Enfermagem. 2008;16(3):445-51.

23. Martínez-Sánchez JM, Fernández E, Fu M, Gallus S, Martínez C, Sureda $X$ et al. Smoking Behaviour, Involuntary Smoking, Attitudes towards Smoke-Free Legislations, and Tobacco Control Activities in the European Union. PLoS ONE. 2010;5(11):e13881.

24. Tan CE, Glantz SA. Association Between Smoke-Free Legislation and Hospitalizations for Cardiac, Cerebrovascular, and Respiratory Diseases: A Meta-Analysis. Circulation. 2012;126: 2177-2183.

25. Córdoba García R. Impacto de la ley del tabaco al primer año de su implantación. Aten Primaria. 2007;39(7):337-8.

26. Marqueta A, Jiménez-Muro A, Beamonte A, Gargallo P, Nerín I. Evolución de la ansiedad en el proceso de dejar de fumar en fumadores que acuden a una Unidad de Tabaquismo. Adicciones. 2010;22(4):317-24.

27. De Granda JI, Carrión F, Alonso S, Márquez FL, Riesco JA, Sampablo I et al. Atención y prestación de servicios en materia de tabaquismo. Arch Bronconeumol. 2006;42(11):600604.

28. Rice VH, Hartmann-Boyce J, Stead LF. Nursing interventions for smoking cessation. Cochrane Database Syst Rev. 2013;8:CD001188. DOI: 10.1002/14651858.CD001188. pub4.

29. Lancaster T, Stead LF. Individual behavioural counselling for smoking cessation. Cochrane Database of Systematic Reviews. 2005, Issue 2. Art. No.: CD001292. DOI: 10.1002/14651858.CD001292.pub2.

30. Torrecilla García M, Barrueco Ferrero M, Maderuelo Fernández JA, Jiménez Ruiz CA, Plaza Martín MD, Hernández Mezquita MA. Deshabituación tabáquica en una consulta de atención primaria: eficacia del consejo médico, la intervención mínima y la terapia sustitutiva con nicotina al año de seguimiento. Aten Primaria. 2001; 27(9): 629-636.

31. Camarelles Guillem F, Dalmau González-Gallarza R, Clemente Jiménez L, Díaz-Maroto Muñoz JL, Lozano Polo A, Pinet Ogué y grupo colaborador Comité Nacional para la Prevención del Tabaquismo (CNPT). Documento de consenso para la atención clínica al tabaquismo en España. Med Clin (Barc). 2013;140(6):272.e1-12. 